Max-Planck-Institut für demografische Forschung

Max Planck Institute for Demographic Research

Doberaner Strasse 114 - D-18057 Rostock · GERMANY

Tel +49 (0) 3812081 - 0; Fax +49 (0) 3812081 - 202;

http://www.demogr.mpg.de

MPIDR WORKING PAPER WP 2001-003

JANUARY 2001

Außerhäusliche Kinderbetreuung in

Ostdeutschland vor und nach der

Wiedervereinigung.

Ein Vergleich mit Westdeutschland in

den Jahren 1990 - 1999

Karsten Hank (hank@ demogr.mpg.de)

Katja Tillmann (katja-tillmann@boeckler.de)

Gert G. Wagner (gwagner@diw.de)

This working paper has been approved for release by: Hans-Peter Kohler (kohler@ demogr.mpg.de)

Head of the Research Group on Social Dynamics and Fertility

(C) Copyright is held by the authors.

Working papers of the Max Planck Institute for Demographic Research receive only limited review. Views or opinions expressed in working papers are attributable to the authors and do not necessarily reflect those of the Institute. 


\title{
Außerhäusliche Kinderbetreuung in Ostdeutschland vor und nach der Wiedervereinigung. Ein Vergleich mit Westdeutschland in den Jahren 1990 - 1999
}

\author{
Karsten Hank $^{*}$, Katja Tillmann ${ }^{* *}$ und Gert G. Wagner ${ }^{* * *}$
}

\begin{abstract}
Zusammenfassung: Im vorliegenden Papier wird zunächst ein kurzer Überblick über Umfang und Ausgestaltung der außerhäuslichen Kinderbetreuung in der ehemaligen DDR, sowie über institutionelle und infrastrukturelle Veränderungen seit der Wiedervereinigung gegeben. Die faktische Betreuungssituation in Ostdeutschland lebender Kinder in den Jahren 1990 bis 1999 wird anschließend auf der Grundlage von Daten des Sozio-oekonomischen Panels (SOEP) untersucht. Das Papier schließt mit Überlegungen zu den Konsequenzen einer sich verändernden Betreuungslandschaft für die Möglichkeiten der Erwerbsbeteiligung von Frauen (insbesondere Müttern) in den neuen Bundesländern.
\end{abstract}

Schlagworte: außerhäusliche Kinderbetreuung, Erwerbsbeteiligung von Frauen, Ostdeutschland

\begin{abstract}
This paper provides first a concise overview of institutional day-care arrangements and their extent in the former GDR. Then institutional and infrastructural changes since German re-unification in 1990 are briefly discussed. In the empirical part, data from the German Socio-Economic Panel (GSOEP) for the period 1990 to 1999 are used to analyze the actual day-care situation for children living in eastern Germany. The paper concludes with considerations of the consequences of a changing day-care situation for the opportunities of women (particularly mothers) in the New Länder to participate in the labor force.
\end{abstract}

Keywords: institutional day-care, labor force participation of women, East Germany

\footnotetext{
* Max-Planck-Institut für demografische Forschung, Doberaner Str. 114, 18057 Rostock. Telefon: 0381-2081-163. Fax: 0381-2081-463.Email: hank@ demogr.mpg.de.

** Wirtschafts- und Sozialwissenschaftliches Institut in der Hans Böckler Stiftung, Düsseldorf.

*** Deutsches Institut für Wirtschaftsforschung, Berlin, und Europa-Universität Viadrina, Frankfurt/Oder.
} 


\section{$\underline{1 . \text { Einleitung }}$}

Die umfassende außerhäusliche Betreuung von Kindern in der ehemaligen DDR ist in der Öffentlichkeit wohlbekannt und in der einschlägigen Literatur bereits gut aufgearbeitet (z.B. Baske 1991; Deutscher Bundestag 1991; Grandke 1990; Hildebrandt 1994; Hille 1988; Mittelbach 1994). Eine solche Versorgung mit Kinderbetreuungsmöglichkeiten sollte neben der frühzeitigen Vermittlung ideologischer Werte (vgl. z.B. Liegle 1988; Schiele 1982) insbesondere dazu beitragen, die Erwerbstätigkeit von Müttern fördern, und so den Arbeitskräftebedarf der Wirtschaft zu befriedigen (z.B. Kistler et al. 1993a; Böckmann-Schewe et al. 1995). Im Gegensatz hierzu wurde und wird in Westdeutschland die Betreuungs- und Erziehungsaufgabe in erster Linie der Familie, d.h. üblicherweise den Müttern, überantwortet. Noch heute erschwert die Ausgestaltung der institutionellen Kinderbetreuung in Westdeutschland eine größere Erwerbsbeteiligung von Müttern (vgl. Kreyenfeld \& Hank (2000) für eine aktuelle Analyse). In diesem Zusammenhang sind in erster Linie die mangelnden Betreuungsmöglichkeiten für Kinder im Krippen- und Hortalter, sowie die restriktiven Öffnungszeiten der Kindergärten zu nennen. ${ }^{1}$

Das ostdeutsche Kinderbetreuungssystem ist eine der auch in den alten Bundesländern vieldiskutierten und häufig als erhaltenswert betrachteten „sozialen Errungenschaften“ aus der Zeit vor der Wende (z.B. Sternitzky und Putzing 1996; Schmidt 1990). Die großen Unterschiede zwischen der ehemaligen DDR und der alten Bundesrepublik hinsichtlich der Versorgung mit Einrichtungen zur außerhäuslichen

\footnotetext{
${ }^{1}$ Für eine ausführliche Analyse und Kritik der derzeitigen Organisation institutioneller Kinderbetreuung in Deutschland vgl. Kreyenfeld et al. 2000.
} 
Kinderbetreuung werfen jedoch die Frage nach der diesbezüglichen Entwicklung in Ostdeutschland seit der Wiedervereinigung auf. In diesem Papier wird zunächst ein kurzer Überblick über Umfang und Ausgestaltung der außerhäuslichen Kinderbetreuung in der ehemaligen DDR (Abschnitt 2), sowie über institutionelle und infrastrukturelle Veränderungen seit der Wiedervereinigung ( Betreuungssituation in Ostdeutschland lebender Kinder, d.h. die tatsächliche Inanspruchnahme außerhäuslicher Kinderbetreuung in den Jahren 1990 bis 1999, wird in $\underline{\text { Abschnitt } 4}$ auf der Grundlage von Daten des Sozio-oekonomischen Panels (SOEP) untersucht. Das Papier schließt mit Überlegungen zu den Konsequenzen einer sich verändernden Betreuungslandschaft für die Möglichkeiten der Erwerbsbeteiligung von Frauen (insbesondere Müttern) in den neuen Bundesländern (Abschnitt 5).

\section{Umfang und Ausgestaltung der außerhäuslichen Betreuung von Kindern}

\section{in der ehemaligen DDR}

In der DDR existierte eine Vielzahl familien- und sozialpolitischer Maßnahmen, die insbesondere auf die Förderung der Vereinbarkeit von Erwerbstätigkeit und Kindererziehung abzielten (für einen Überblick vgl. z.B. Frerich \& Frey 1996; Wendt 1997). Neben monetären Transfers, die einen erheblichen Teil der durch Kinder verursachten Kosten ausgeglichen haben (z.B. Ott et al. 1990), spielte hierbei die Betreuung von Kindern in Tageseinrichtungen eine herausragende Rolle (z.B. Grandke 1990).

Seit Gründung der DDR wurde die außerhäusliche Betreuung von Vorschul- und Schulkindern staatlich gefördert, sodass in den 80er Jahren ein nahezu flächendeckendes 
Angebot an Betreuungseinrichtungen für Kinder im Vor- und Grundschulalter zur Verfügung stand (vgl. z.B. Hildebrandt 1994). Bereits mit Beginn der 80er Jahre stand für alle Kinder deren Eltern es wünschten ein Krippen-, Kindergarten- oder Hortplatz zur Verfügung. In der DDR lagen 1989 die amtlichen Betreuungsquoten für Krippen bei circa $80 \%$, für Kindergärten bei etwa 95\%, und für Schulhorte für Grundschüler bei gut 80\% (vgl. Frerich \& Frey 1996).

Die Öffnungszeiten der Kindertageseinrichtungen waren den Arbeitszeiten der Mütter angepaßt. Kindergärten und Krippen öffneten gegen $6.00 \mathrm{Uhr}$ morgens und waren dann durchgehend bis etwa 18.00 Uhr geöffnet. Im Bedarfsfall (z.B. bei Teilnahme der Eltern an Weiterbildungsmaßnahmen) konnte eine Betreuung auch samstags erfolgen. Schulhorte waren auch in den Ferien ganztägig geöffnet (vgl. Schmidt 1992). Die Kosten, die die Eltern für die Ganztagsbetreuung ihrer Kinder aufbringen mußten, waren minimal. Die Beteiligung an den Verpflegungskosten betrug pro Tag 1,40 Mark für die Verpflegung von Krippenkindern, 0,35 Mark für ein Mittagessen im Kindergarten und 0,55 Mark für ein Mittagessen im Schulhort (vgl. Ott et al. 1990). Die Unterbringung der Kinder in Tageseinrichtungen war für die Familien damit billiger als die häusliche Versorgung.

Es bestand keine Pflicht für Eltern, ihre Kinder in einer Einrichtung betreuen zu lassen. Faktisch war aber der Alltag für Familien (und insbesondere für die zum größten Teil vollerwerbstätigen Mütter $^{2}$ ) ohne die außerhäusliche Betreuung ihrer Kinder nicht zu bewältigen. Teilzeitarbeit war als „Schonarbeitplatz“ eher für ältere Frauen

2 Vgl. z.B. Böckmann-Schewe et al. (1995) zur traditionellen Rollenzuweisung im Reproduktionsbereich bei gleichzeitiger formaler Gleichstellung der Frauen im Berufsleben der DDR. 
vorgesehen und für Mütter kaum realisierbar (z.B. Schupp 1991). Eine realistische Alternative zur Kinderbetreuung in staatlichen Einrichtungen existierte nicht. In der DDR befand sich der überwiegende Teil der Kinderbetreuungseinrichtungen in staatlicher Trägerschaft. Der Anteil der Einrichtungen in betrieblicher Trägerschaft betrug immerhin noch 12\%, während die Kirchen mit lediglich 164 Einrichtungen praktisch keine Rolle spielten (vgl. Deutscher Bundestag 1991).

\section{$\underline{\text { 3. Die Versorgung mit außerhäuslicher Kinderbetreuung in Ostdeutschland }}$}

\section{nach der Wiedervereinigung}

Als Konsequenz des föderalen Aufbaus der Bundesrepublik Deutschland ging nach der Wiedervereinigung die finanzielle Verantwortung für die staatlichen Tageseinrichtungen für Kinder auf die ostdeutschen Kommunen über (vgl. auch Sternitzky und Putzing (1996) zum Wandel der Eigentums und Trägerstrukturen). Dies führte in den Folgejahren zu erheblichen Finanzierungsproblemen ${ }^{3}$, die sich insbesondere negativ auf die Versorgung mit Schulhorten ausgewirkt haben. Dennoch lag 1994 der Umfang der Betreuungsangebote für Kinder aller Altersklassen in den neuen Bundesländern immer noch weit über den jeweiligen Versorgungsquoten in Westdeutschland (siehe Tabelle 1). Dies gilt insbesondere für die Ganztagsbetreuung in Kindergärten und die Betreuung der 0 bis 3jährigen in Krippen, sowie der 6 bis 10jährigen in Horten. Obwohl Schulhorte nicht mehr selbstverständlicher Teil ostdeutscher Schulen sind, liegt die Versorgungsquote hier immerhin noch bei $60 \%$. In Westdeutschland sind hingegen

\footnotetext{
${ }^{3}$ Eine aktuelle Diskussion bestehender und alternativer Finanzierungsmodelle institutioneller Kinderbetreuung findet sich in Kreyenfeld et al. 2000.
} 
Angebote zur Nachmittagsbetreuung von Schulkindern bislang ebenso wenig üblich, wie die Existenz von Ganztagsschulen (vgl. hierzu Flehmig 1995).

[Tabelle 1 etwa hier einfügen]

In der Vergangenheit wurde der deutliche Rückgang der Erwerbstätigkeit unter ostdeutschen Frauen häufig u.a. mit einem Rückgang der Betreuungsmöglichkeiten erklärt (vgl. z.B. Kistler et al. 1993a, 1993b). Es mag daher überraschen, dass trotz finanzieller Engpässe bei den Kommunen und einem starken Abbau der Plätze in Betreuungseinrichtungen die Versorgungsquoten immer noch so hoch sind, wie in Tabelle 1 dargestellt. Man muß in diesem Zusammenhang allerdings berücksichtigen, dass sich die Geburtenraten in den neuen Bundesländern gegenüber den in der ehemaligen DDR beobachteten Werten zwischenzeitlich mehr als halbiert hatten (vgl. Kreyenfeld 2000, Witte \& Wagner 1995). ${ }^{4}$ Um die Veränderungen in der ostdeutschen Betreuungslandschaft seit der Wiedervereinigung offenzulegen, erscheint somit eine Analyse sinnvoll, die nicht allein auf einer Untersuchung des Angebotes von Kinderbetreuungseinrichtungen beruht (z.B. Sternitzky \& Putzing 1996), sondern auch die tatsächliche Inanspruchnahme außerhäuslicher Kinderbetreuung durch Privathaushalte berücksichtigt.

\footnotetext{
${ }^{4}$ Vgl. auch Schulz (1994) zu Auswirkungen des Bevölkerungsrückgangs in den neuen Ländern auf ausgewählte Infrastrukturbereiche.
} 


\section{Empirische Analyse der faktischen Betreuungssituation in}

Ostdeutschland, 1990 bis 1998

\subsection{Datengrundlage}

Datenbasis der vorliegenden Untersuchung ist das Sozio-oekonomische Panel (SOEP) des Deutschen Instituts für Wirtschaftsforschung, Berlin. Das SOEP wurde 1984 als jährliche Wiederholungsbefragung deutscher und ausländischer Privathaushalte in Westdeutschland gestartet (vgl. Projektgruppe Sozio-oekonomisches Panel 1995). Bereits im Juni 1990 wurde das SOEP auf die damalige DDR ausgeweitet. Seit 1991 findet jeweils im Frühjahr in Ost- und Westdeutschland die Erhebung für das SOEP statt. Der Umfrage wurde 1994/95 eine Zuwandererstichprobe und 1998 eine Ergänzungsstichprobe hinzugefügt. Im vorliegenden Beitrag werden nur Privathaushalte der Stichproben A (westdeutscher Haushaltsvorstand) und C (ostdeutscher Haushaltsvorstand) berücksichtigt. ${ }^{5}$

Die Haushaltsstichprobe des SOEP ermöglicht „kinderbezogene“ Analysen auf der Grundlage von Angaben der jeweiligen Hauptauskunftsperson über die Kinder im Haushalt. Damit kann untersucht werden, ob ein Kind außerhäuslich betreut wird, bzw. welche Schulart es besucht. Die folgende Analyse bezieht sich auf den Zeitraum Juni 1990 bis zur aktuellen Welle des SOEP für das Jahr 1999. Es ist plausibel anzunehmen, dass die Situation im Juni 1990 noch weitgehend der in der ,alten DDR“ entsprach: weder die Erwerbsquoten hatten sich zu diesem Zeitpunkt verändert, noch die

\footnotetext{
${ }^{5}$ Vgl. Binder (1995) für eine Analyse der unterschiedlichen Inanspruchnahme außerhäuslicher Betreuung durch deutsche und ausländische Kinder (in Westdeutschland).
} 
institutionellen Rahmenbedingungen. Insbesondere die betrieblichen und staatlichen Subventionen im sozialen Bereich entsprachen noch weitgehend den alten DDRRegelungen.

Für den Vergleich der Betreuungssituation unmittelbar vor der Wirtschafts-, Währungs- und Sozialunion mit der Betreuungssituation neun Jahre nach der Wende in Ostdeutschland stellt das SOEP eine nahezu einmalige, jedoch nicht gänzlich unproblematische Datenbasis dar. Im Laufe der 1990er Jahre wurden die Betreuungsfragen im SOEP zweimal (1992 und 1995) umformuliert. $^{6}$ Dies bedeutet zwar im Allgemeinen kein großes Problem hinsichtlich der Vergleichbarkeit der Betreuungsquoten über die Zeit. Die Betreuung von Grundschülern in Horten wird in den Jahren 1992 - 1994 jedoch systematisch unterschätzt, sodass die für diese Gruppe in Tabelle 2 und Tabelle 3 ausgewiesenen Betreuungsquoten nicht sinnvoll interpretiert werden können.

\subsection{Ergebnisse}

Die in Tabelle 2 dargestellten deskriptiven Ergebnisse zeigen, dass sich die Betreuungsquoten für ostdeutsche Kinder im Krippenalter kurz vor und kurz nach der Wiedervereinigung kaum voneinander unterschieden haben. Sowohl im Juni 1990 als auch im Frühjahr 1992 wurden ca. 60\% der 0 bis 3jährigen mindestens halbtags außerhäuslich betreut. Ab 1993 ging die außerhäusliche Betreuung von Kindern dieser Altersgruppe jedoch deutlich auf etwa die Hälfte des früheren DDR-Niveaus zurück. In

\footnotetext{
${ }^{6} \mathrm{Vgl}$. hierzu die unter http://www.diw.de/deutsch/sop/soepinfo/ online verfügbaren Fragebögen der jeweiligen Wellen.
} 
den späten 90er Jahren pendelte sich die Betreuungsquote für 0 bis 3jährige dann bei etwa $30 \%$ ein. $^{7}$

Die außerhäusliche Betreuung von Kindergartenkindern geht im Beobachtungszeitraum relativ kontinuierlich, aber moderat zurück. Ausgehend von einer Betreuungsquote unter 4 bis 6jährigen von $98 \%$ im Jahr 1990, sank diese auf 89\% neun Jahre später. Damit hat der Anteil außerhäuslich betreuter Kinder dieser Altersklasse in etwa das westdeutsche Niveau erreicht.

Sehr ähnliche Schätzungen für die Betreuung von Kindern im Vorschulalter zu Beginn der 90er Jahre finden sich auch in anderen Studien: Höckner (1995) weist z.B. für das Jahr 1990 eine Betreuungsquote für 3 bis 6jährige von 94\% aus (für 1 bis 2jährige: 80\%). Bei Joos und Nauck (1996) ergeben sich für 3 bis 6 Jahre alte Kinder Betreuungsquoten von $94 \%$ und für bis unter 3jährige Kinder von 58\% in 1990. Für das Jahr 1993 finden Joos und Nauck Betreuungsquoten von 83\% für die 3 bis 6jährigen und von 39\% für die unter 3jährigen.

Damit liegen die Betreuungsquoten für Krippenkinder, die auf der Basis von nach der Wiedervereinigung erhobenen Surveys berechnet wurden, deutlich unter denen der amtlichen Statistik der DDR (vgl. Abschnitt 2). Dies gilt auch für die Betreuung von Grundschülern in Horten. Das SOEP weist hier für 1990 eine Quote von 35\% aus. ${ }^{8}$ Hier

\footnotetext{
${ }^{7}$ Die Unterschiede zwischen den Jahren 1997 bis 1999 in Tabelle 2 sind aufgrund der niedrigen Fallzahlen in der Stichprobe statistisch nicht signifikant.

${ }^{8}$ Eine Untersuchung mit Daten des Mikrozensus für das Jahr 1996 zeigt, dass bereits kleine Änderungen bei der Abgrenzung der Altersklassen zu erheblichen Unterschieden in den geschätzten Betreuungsquoten führen können (vgl. hierzu Kreyenfeld \& Spieß 2001). Die vorliegende Analyse basiert zwar im Wesentlichen auf Angaben für 7 bis 11jährige, schließt
} 
hat unerwarteterweise kein deutlicher Rückgang stattgefunden: auch in der Mitte und am Ende der 1990er Jahre wurden noch zwischen 25 und 30\% der 7 bis 11 jährigen außerhäuslich betreut.

Auch nach der Wiedervereinigung blieb die Bedeutung außerhäuslicher Betreuung von Krippenkindern und Grundschülern in Ostdeutschland um ein Vielfaches höher als im Westen. Hier werden immer noch typischerweise nur die 4 bis 7 jährige Vorschulkinder betreut (vgl. hierzu auch Binder 1995). Die Betreuungsquoten für Kinder aller Altersgruppen blieben in Westdeutschland in den 1990er Jahren nahezu konstant (Krippe: 6\%, Kindergarten: $80-85 \%$, Hort: 3\%).

[Tabelle 2 etwa hier einfügen]

Ein ähnliches Bild wie für die Betreuungssituation insgesamt zeigt sich auch, wenn die Entwicklung der Ganztagsbetreuungsquoten betrachtet wird (Tabelle 3). Im Falle von Grundschülern (also den 7 bis 11jährigen) entspricht die Ganztagsbetreuungsquote der in Tabelle 2 ausgewiesenen Betreuungsquote, da angenommen wird, dass eine nachmittägliche Betreuung von Schülern im Endergebnis einer Ganztagsbetreuung gleichkommt.

Während bis 1992 noch etwa 45 bis 50\% der bis zu 3jährigen ostdeutschen Kinder ganztägig außerhäuslich betreut wurden, reduzierte sich dieser Anteil danach deutlich,

jedoch auch jene 6 und 12jährigen ein, die bereits, bzw. immer noch eine Grundschule besuchen. 
bis auf weniger als ein Viertel der Kinder in der entsprechenden Altersgruppe. ${ }^{9}$ Auch bei der Ganztagsbetreuung von Kindergartenkindern zeigte sich in der zweiten Hälfte der 90er Jahre ein deutlicher Rückgang: wurden 1990 noch 80\% und bis 1994 noch ca. 70\% der 4 bis 7jährigen Ostdeutschen ganztags betreut, waren es ab 1996 nur noch etwa 50\%. Allerdings liegt damit die Ganztagsbetreuungsquote für Kinder dieser Altersklasse in den neuen Ländern immer noch um das Zwei- bis Dreifache über derjenigen in Westdeutschland.

[Tabelle 3 etwa hier einfügen]

\section{$\underline{\text { 5. Diskussion }}$}

Hinsichtlich des Zusammenhangs zwischen der außerhäuslichen Betreuung von Kindern in Ostdeutschland und den Möglichkeiten der Erwerbsbeteiligung von Frauen in den neuen Bundesländern, lassen sich zwei Hauptergebnisse der hier durchgeführten Untersuchung festhalten:

Erstens ist ein teilweise deutlicher Rückgang der Betreuungsquoten für Kinder aller Altersgruppen nach der Wiedervereinigung festzustellen. Gleichwohl liegen insbesondere die für eine Vollzeiterwerbstätigkeit von Müttern wichtigen Ganztagsbetreuungsquoten immer noch um ein Vielfaches über dem westdeutschen Niveau. Dies dokumentiert zum einen unterschiedliche Einstellungen in Ost und West zur Vereinbarkeit von Mutterschaft und Erwerbsarbeit durch die Inanspruchnahme

\footnotetext{
${ }^{9}$ Die Fallzahlen im SOEP sind hier extrem klein, sodass eine genauere Interpretation der Werte in Tabelle 3 sinnvoll nicht möglich ist.
} 
außerhäuslicher Kinderbetreuung auch für Kleinkinder (vgl. z.B. Dannenbeck et al. 1995; Habich \& Noll 1997). Zum anderen weist es auf die im Vergleich zur ehemaligen DDR zwar schlechteren, im Vergleich zu Westdeutschland jedoch besseren Möglichkeiten einer solchen Vereinbarkeit hin.

Diese Möglichkeiten wurden jedoch angesichts der Lage auf dem ostdeutschen Arbeitsmarkt in den 1990er Jahren nicht immer genutzt. Daher lässt sich zweitens feststellen, dass die in Tabelle 1 ausgewiesenen Versorgungs-Quoten über den ermittelten Betreuungs-Quoten in Tabelle 2 und Tabelle 3 liegen. ${ }^{10}$ Diese Beobachtung deckt sich mit den Befunden anderer Untersuchungen (z.B. Sternitzky und Putzing 1996), die ebenfalls - regional unterschiedlich stark ausgeprägte - Überkapazitäten konstatieren. Es ist davon auszugehen, dass neben der Inanspruchnahme des seit 1992 auf drei Jahre verlängerten Erziehungsurlaubs insbesondere die hohe Frauenerwerbslosigkeit in Ostdeutschland $\mathrm{zu}$ einer Verlagerung der Bedeutung außerhäuslicher Kinderbetreuung zugunsten einer Betreuung durch die Mütter geführt hat (vgl. Engelbrech \& Jungkunst 1998). Daher ist zu befürchten, dass es zu einem weiteren Abbau der Plätze in Tageseinrichtungen für Kinder kommen wird. Bei einer Besserung der Lage auf dem Arbeitsmarkt wären dann auch in Ostdeutschland die für eine entsprechende Erwerbsbeteiligung von Müttern notwendigen außerhäuslichen Betreuungsmöglichkeiten nicht mehr in ausreichendem Maße vorhanden.

\footnotetext{
${ }^{10}$ Es sei an dieser Stelle darauf hingewiesen, dass beide Quoten nicht direkt miteinander vergleichbar sind. Während die Versorgungsquoten auf prozeßproduzierten Daten der kommunalen Verwaltungen beruhen, wurden die Versorgungsquoten auf Basis einer Befragung mit teilweise sehr kleinen Fallzahlen berechnet. Beide Datengrundlagen haben Vor- und Nachteile und weisen ein spezifisches Fehlerpotenzial auf. Dies ändert jedoch nichts an der allgemeinen Aussage, die sich aus einem Vergleich beider Quoten ableiten läßt.
} 


\section{$\underline{\text { Literatur }}$}

Baske, Siegfried (1991): Pädagogik und Bildungswesen in der ehemaligen DDR, in: Roth, Leo (Hg.), Pädagogik: Handbuch für Studium und Praxis, München, S.563571.

Binder, Marion, (1995): Soziostrukturell differenzierte Inanspruchnahme außerhäuslicher Betreuung von Kindern im Vorschulalter - eine empirische Analyse für die Bundesrepublik Deutschland (alte Bundesländer) für das Jahr 1993, in: Zeitschrift für Familienforschung, 7 (2), S.89-118.

Böckmann-Schewe, Lisa \& Christine Kulke \& Anne Röhrig (1995): „Es war immer so, den goldenen Mittelweg zu finden zwischen Familie und Beruf war eigentlich das Entscheidende.“ Kontinuitäten und Veränderungen im Leben von Frauen in den neuen Bundesländern, in: Berliner Journal für Soziologie, 5 (2), S.207-222.

Dannenbeck, Clemens \& Sabrina Keiser \& Tatjana Rosendorfer (1995): Familienalltag in den alten und neuen Bundesländern - Aspekte der Vereinbarkeit von Beruf und Familie, in: Bernhard Nauck et al. (Hg.), Familie und Lebensverlauf im gesellschaftlichen Umbruch, Stuttgart, S.103-118.

Deutscher Bundestag (1991): Situation der Kindergärten, Krippen und Horte in den neuen Bundesländern, Bundestagsdrucksache 12/661.

Deutsches Jugendinstitut (1998): Tageseinrichtungen für Kinder. Pluralisierung von Angeboten. Zahlenspiegel, München.

Grandke, A. (1990): Zur Familienpolitik der DDR - Fragen der künftigen Familienpolitik in Deutschland, in: Deutschalnd-Archiv, 23 (6), S.863-868.

Engelbrech, Gerhard \& Maria Jungkunst (1998): Erwerbsbeteiligung von Frauen und Kinderbetreuung in ost- und westdeutschen Familien, IAB Werkstattbericht 2/98.

Flehmig, Susanne (1995): Institutionelle Grundlagen der Betreuung von Schulkindern in Deutschland, Diskussionspapier aus der Fakultät für Sozialwissenschaft der RuhrUniversität Bochum Nr. 95-16, Bochum. 
Frerich, Johannes \& Martin Frey (1996): Handbuch der Geschichte der Sozialpolitik in Deutschland, Band 2: Sozialpolitik in der Deutschen Demokratischen Republik, München und Wien.

Habich, Roland \& Heinz-Herbert Noll (Hg.) (1997): Objektive Lebensbedingungen und subjektives Wohlbefinden im vereinten Deutschland, in: Statistisches Bundesamt (Hg.), Datenreport 1997, Bonn.

Hildebrandt, Regine (1994): Die Einrichtungen des Gesundheits- und Sozialwesens in der DDR und in den neuen Bundesländern, in: Aus Politik und Zeitgeschichte, B3, S.15-26.

Hille, Barbara (1988): Kinder in der DDR - Betreuung und Erziehung in der frühen Kindheit und im Vorschulalter, in: Deutschland-Archiv, 21, S.513-526.

Höckner, Marianne (1995): Der Staat hat viele Väter - wo bleiben die Mütter?, in: Bernhard Nauck \& Hans Bertram (Hg.), Kinder in Deutschland, Opladen, S.333356.

Joos, Magdalena \& Bernhard Nauck (1996): Wandel der familiären Lebensverhältnisse von Kindern in Ostdeutschland, Expertise für die Berichtsgruppe 5 der KSPW, Chemnitz.

Kistler, Ernst \& Dieter Jaufmann \& Anita B. Pfaff, (1993a): „Die Wiedervereinigung der deutschen Männer braucht keine Frauen..." - Frauen als Wendeverliererinnen?, in: Aus Politik und Zeitgeschichte, B6, S.39-52.

Kistler, Ernst \& Anita B. Pfaff \& Dieter Jaufmann (1993b): Aktuelle Daten zur Entwicklung der Kindereinrichtungen in den neuen Ländern. Es droht eine Abwärtsspirale, in: Arbeit und Sozialpolitik 40 (3-4), S.49-54.

Kreyenfeld, Michaela (2000): Changes in the Timing of First Birth in East Germany after Re-Unification, in: Schmollers Jahrbuch, 120 (2), S.169-186.

Kreyenfeld, Michaela \& Karsten Hank (2000): Does the availability of child care influence the emplyoment of mothers? Findings from western Germany, in: Population Research and Policy Review, 19 (4), S.317-337.

Kreyenfeld, Michaela \& C. Katharina Spieß \& Gert G. Wagner (2000): Finanzierungsund Organisationsmodelle institutioneller Kinderbetreuung, Neuwied. 
Kreyenfeld, Michaela \& C. Katharina Spieß (2001): Finanzierung und Rationierung von Kinderbetreuungsplätzen in Deutschland, mimeo, Rostock und Berlin.

Liegle, Ludwig (1988): Erziehung zur Anpassung. Das Beispiel der Deutschen Demokratischen Republik (DDR), in: Neue Sammlung, 28, S.35-48.

Mittelbach, Hans (1994): Familienpolitik und Lage der Familien in den neuen Bundesländern, in: Jürgen Zerche (Hg.), Vom sozialistischen Versorgungsstaat zum Sozialstaat Bundesrepublik. Ausbau oder Abbau der sozialen Lage in den neuen Bundesländern?, Regensburg, S.55-95.

Ott, Notburga \& Heidrun Radtke \& Wera Thiel \& Gert Wagner (1990): Kindererziehung und Erwerbsarbeit - marktwirtschaftliche Möglichkeiten einer erziehungsfreundlichen Erwerbsarbeit in Deutschland, in: Wirtschaftswissenschaft (DDR), 38 (9), S.1242-1261.

Projektgruppe Sozio-oekonomisches Panel (1995): Das Sozio-oekonomische Panel (SOEP) im Jahre 1994, in: Vierteljahrshefte zur Wirtschaftsforschung, 64 (1), S.513.

Schiele, Eckart (1982): Wehrerziehung im Kindergarten. Zur Problematik der Schaffung eines eigenständigen Nationalgefühls, in: Pädagogik und Schule in Ost uns West, 31, 1982, 2, S.27-35.

Schmidt, Franz-Hermann (1993): Gedanken zur Horterziehung in der SBZ/DDR. Ansätze für eine Periodisierung, in: Pädagogische Führung - Zeitschrift für Schulleitung und Schulberatung, 4, 1993, 3, S.132-134.

Schmidt, Hans-Dieter (1990): Wo Kehrtmarsch-Ideologen reden, mische ich mich ein. Kinderkrippen in der DDR - Was war gut und was war schlecht, und welche Reformen sind jetzt die wichtigsten?, in: Neues Deutschland vom 23./24.Juni 1990, S.10.

Schulz, Erika (1994): Auswirkungen des Bevölkerungsrückganges in den neuen Bundesländern auf ausgewählte Infrastrukturbereiche, in: Vierteljahreshefte zur Wirtschaftsforschung, S.491-506.

Schupp, Jürgen (1991): Teilzeitarbeit in der DDR und in der Bundesrepublik Deutschland, in: Projektgruppe Panel (Hg.), Lebenslagen im Wandel - Basisdaten 
und -analysen zur Entwicklung in den neuen Bundesländern, Frankfurt und New York, S.260-279.

Sternitzky, Anita \& Monika Putzing (1996): Kollabiert die Kinderversorgung? - Soziale Einrichtungen im Wandel, in: Hans Bertram (Hg.), Regionen im Vergleich: gesellschaftlicher Wandel in Ostdeutschland am Beispiel ausgewählter Regionen, Opladen, S.17-39.

Wendt, Hartmut (1997): The Former German Democratic Republic: the Standardized Family, in: Franz-Xaver Kaufmann et al. (Hg.), Family Life and Family Policies in Europe, Volume 1, Oxford, S.114-154.

Witte, James C. \& Gert G. Wagner (1995): Declining Fertility in East Germany After Unification: A Demographic Response to Socioeconomic Change, in: Population and Development Review, 21 (2), S.387-397.

\section{Tabellenanhang}

Tabelle 1: Versorgung mit Tageseinrichtungen für Kinder verschiedener Altersgruppen in Ost- und Westdeutschland 1994 - Anzahl der Plätze je 100 Kinder der Altersgruppe

\begin{tabular}{|l|c|c|}
\hline & Ostdeutschland & Westdeutschland \\
\hline Krippe (0-3) & 41 & 2 \\
\hline Kindergarten (3-6) & 116 & 85 \\
\hline Kindergarten (3-6), ganztags - inkl. Mittagessen & 97 & 17 \\
\hline Hort (6-10) & 60 & 5 \\
\hline
\end{tabular}

Quelle: Deutsches Jugendinstitut 1998 
Tabelle 2: Außerhäusliche Betreuung von Vorschul- und Grundschulkindern in Ost- und Westdeutschland in \% der jeweiligen Altersgruppe, 1990 - 1999

\begin{tabular}{|c|c|c|c|c|c|c|c|c|c|c|c|c|c|}
\hline \multirow{2}{*}{$\begin{array}{l}\text { Alter d. } \\
\text { Kinder }\end{array}$} & \multicolumn{10}{|c|}{ Ostdeutschland } & \multicolumn{3}{|c|}{ Westdeutschland } \\
\hline & 1990 & 1991 & 1992 & 1993 & 1994 & 1995 & 1996 & 1997 & 1998 & 1999 & 1990 & 1995 & 1999 \\
\hline $0-3$ & 62 & 63 & 57 & 50 & 30 & $21^{\mathrm{c})}$ & $23^{c)}$ & 33 & 27 & 34 & $6^{c)}$ & $6^{c)}$ & $7^{c)}$ \\
\hline $4-6^{\mathrm{a})}$ & 98 & 94 & 93 & 91 & 90 & 92 & 88 & 87 & 85 & 89 & 82 & 80 & 85 \\
\hline $7-11^{\mathrm{b})}$ & 35 & 31 & $12^{f)}$ & $16^{\mathrm{f})}$ & $15^{\mathrm{f})}$ & 27 & 30 & 27 & 30 & 24 & $2^{\mathrm{e})}$ & $3^{\text {d) }}$ & $5^{\mathrm{d})}$ \\
\hline $\begin{array}{l}\text { a) Inkl. } 7 \\
\text { b) Inkl. } 6 \\
\text { c) Zellen } \\
\text { d) Zellen } \\
\text { d) Zellen } \\
\text { f) Wert n }\end{array}$ & $\begin{array}{l}\text { hrige, } \\
\text { ind } 12 \\
\text { esetzu } \\
\text { esetzu } \\
\text { esetzu } \\
\text { ht sin }\end{array}$ & $\begin{array}{l}\text { noch } 1 \\
\text { ige, di } \\
\text { n der } S \\
\text { n der } S \\
\text { der } S \\
11 \text { inter }\end{array}$ & $\begin{array}{l}\text { d die G } \\
\text { ereits, b } \\
\text { hprobe } \\
\text { hprobe } \\
\text { hprobe } \\
\text { tierbar }\end{array}$ & $\begin{array}{l}\text { idschul } \\
\text { inmer als } \\
\text { iner als } \\
\text { iner als } \\
\text { 1. Absc }\end{array}$ & $\begin{array}{l}\text { esuchen } \\
\text { och die } \\
\text { ). } \\
\text { itt 4.1). }\end{array}$ & undsch & besuch & & & & & & \\
\hline
\end{tabular}

Quelle: SOEP Welle 6 - 16, eigene Berechnungen 
Tabelle 3: Ganztagsbetreuungsquoten für Vorschul- und Grundschulkinder in Ost- und Westdeutschland in \% der jeweiligen Altersgruppe,

$1990-1999$

\begin{tabular}{|c|c|c|c|c|c|c|c|c|c|c|c|c|c|}
\hline \multirow{2}{*}{$\begin{array}{l}\text { Alter } d \text {. } \\
\text { Kinder }\end{array}$} & \multicolumn{10}{|c|}{ Ostdeutschland } & \multicolumn{3}{|c|}{ Westdeutschland } \\
\hline & 1990 & 1991 & 1992 & 1993 & 1994 & 1995 & 1996 & 1997 & 1998 & 1999 & 1990 & 1995 & 1999 \\
\hline $0-3$ & 52 & 43 & 46 & 38 & $22^{c)}$ & $14^{\mathrm{d})}$ & $12^{\mathrm{d})}$ & $15^{\mathrm{d})}$ & $14^{\mathrm{d})}$ & $15^{\mathrm{d})}$ & $2^{\mathrm{e})}$ & $1^{\mathrm{e})}$ & $1^{\mathrm{e})}$ \\
\hline $4-6^{a)}$ & 80 & 70 & 72 & 74 & 68 & 60 & 47 & 45 & 51 & 56 & 21 & 15 & 20 \\
\hline $7-11^{\mathrm{b})}$ & 35 & 31 & $12^{\mathrm{f})}$ & $16^{\mathrm{f})}$ & $15^{f)}$ & 27 & 30 & 27 & 30 & 24 & $2^{\mathrm{e})}$ & $3^{\mathrm{d})}$ & $5^{d)}$ \\
\hline
\end{tabular}

a) Inkl. 7jährige, die noch nicht die Grundschule besuchen.

b) Inkl. 6 und 12jährige, die bereits, bzw. immer noch die Grundschule besuchen.

c) Zellenbesetzung in der Stichprobe kleiner als 30 .

d) Zellenbesetzung in der Stichprobe kleiner als 20.

e) Zellenbesetzung in der Stichprobe kleiner als 10.

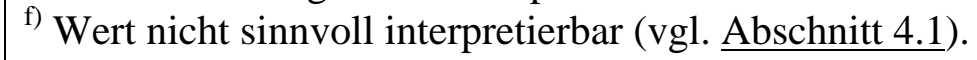

Quelle: SOEP Welle 6 - 16, eigene Berechnungen 